\title{
On the Green's Function of the almost-Mathieu Operator
}

\author{
Florian G Chmela and Gustav M Obermair \\ Universität Regensburg, D- 93040 Regensburg, Germany
}

\begin{abstract}
The square tight-binding model in a magnetic field leads to the almostMathieu operator which, for rational fields, reduces to a $q \times q$ matrix depending on the components $\mu, \nu$ of the wave vector in the magnetic Brillouin zone. We calculate the corresponding Green's function without explicit knowledge of eigenvalues and eigenfunctions and obtain analytical expressions for the diagonal and the first offdiagonal elements; the results which are consistent with the zero magnetic field case can be used to calculate several quantities of physical interest (e. g. the density of states over the entire spectrum, impurity levels in a magnetic field).
\end{abstract}




\section{Introduction}

Different approaches to the problem of an electron in a twodimensional periodic potential with an applied perpendicular magnetic field (see e.g. [1] or [2]) allow the reduction of the twodimensional eigenvalue equation to a one dimensional difference equation of the form

$$
g_{m+1}+g_{m-1}+2 \gamma \cos (2 \pi \alpha m-\nu) g_{m}=\epsilon g_{m},
$$

known as almost-Mathieu or Azbel-Harper equation, where $\epsilon$ is the energy of the electron scaled in units of the bandwidth at zero magnetic field.

Equation (1) corresponds to an effective Hamiltonian

$$
\mathbf{H}_{e f f}=2 \cos \left(\hat{p}_{x}\right)+2 \gamma \cos (2 \pi \alpha \hat{x}-\nu) .
$$

It has been studied extensively ever since Hofstadter's pioneering work [1], both for the fascinating cantor-set properties and selfsimilarities of its eigenvalue spectrum spec $=\left\{\epsilon \mid g_{\mathrm{m}}\right.$ non diverging $\}$ (see e.g. [3], [4], [5] and references therein) and for its significance for periodically structured $2 \mathrm{~d}$-electron systems (e.g. [6] and [7]).

In the following we restrict ourselves to the symmetric case $\gamma=1$ corresponding to square symmetry of the $2 \mathrm{~d}$ periodic potential.

The phase $\nu \in[0,2 \pi]$ in (1) represents the component of the wave vector in $y-$ direction, $\alpha$ is the number of magnetic flux quanta per unit cell or - depending on the chosen ansatz - the number of unit cells per flux quantum.

As in most other work on this problem, we restrict $\alpha$ to the dense set of rational values

$$
\alpha=\frac{p}{q},(p \in \mathbb{Z}, q \in \mathbb{N}) .
$$

With this restriction (11) becomes periodic, i.e it remains invariant under the substitution $m \mapsto m+q$. We therefore may assume $m \in\{0,1, \ldots, q-1\}$. According to Floquet's theorem (1) then has at least one solution of the form

$$
g_{m+q}=\mathrm{e}^{\mathrm{i} q \mu} g_{m} .
$$

The phase $\mu$ can be identified with the wave vector in $x$-direction. With (2) the solution of the almost-Mathieu equation (1) can be reduced to the eigenvalue problem

$$
\mathbf{H} \psi=\epsilon \psi
$$

of the $q$-dimensional matrix corresponding to the almost-Mathieu operator $\mathbf{H}$ for rational $\alpha$ :

$$
\mathbf{H}(\mu, \nu)=\mathbf{H}:=\left(\begin{array}{cccccc}
c_{1} & 1 & 0 & \cdots & 0 & \mathrm{e}^{\mathrm{i} q \mu} \\
1 & c_{2} & 1 & & & 0 \\
0 & 1 & \ddots & & & \vdots \\
\vdots & 0 & & & \ddots & 0 \\
0 & \vdots & & \ddots & \ddots & 1 \\
\mathrm{e}^{-\mathrm{i} q \mu} & 0 & \cdots & 0 & 1 & c_{q}
\end{array}\right),
$$


where $c_{j}:=2 \cos (2 \pi j \alpha-\nu)$.

As has first been shown in [2], the eigenvalues of (4) can be obtained as the roots $\epsilon$ of the equation

$$
\operatorname{det}(\epsilon-\mathbf{H})=P(\epsilon)-2 \cos (q \mu)-2 \cos (q \nu)=0 .
$$

Here $P(\lambda)$ is defined as the polynomial of degree $q$ in $\lambda$ for any $\lambda \in \mathbb{C}$ :

$$
P(\lambda):=\operatorname{det}\left(\lambda-\mathbf{H}\left(\frac{\pi}{2 q}, \frac{\pi}{2 q}\right)\right)=\operatorname{det}(\lambda-\mathbf{H}(0,0))-4 \text {. }
$$

It depends only on $\lambda$ and on $\alpha$, but not on the phases $\mu$ and $\nu$.

According to (5) the spectrum $\operatorname{spec}(\mathbf{H})$ is given by the real solutions of

$$
P(\epsilon)=P(\epsilon(\mu, \nu))=2 \cos (q \mu)+2 \cos (q \nu) .
$$

Evidently for each fixed pair of values $(\mu, \nu)$ (i.e. one point in the magnetic Brillouin zone) (6) yields $q$ real eigenvalues. Varying $\nu \in[-\pi, \pi]$ and $\mu \in[-\pi, \pi]$, the eigenvalues are broadened into $q$ magnetic subbands $\epsilon(\mu, \nu)$ [1]: each eigenvalue $\epsilon$ is a function of the momenta $\nu$ and $\mu$ and of the subband-index $k$, with $1 \leq k \leq q$. Except for some special cases where two subbands share one common point, e.g. for even $q$ at $\epsilon=0$, these subbands are disjoint. The graph of this subband structure in dependence on $\alpha$ is known as Hofstadter's Butterfly [1].

According to (4), the eigenvectors $\psi_{k}$ in (3) are vectors in $\mathbb{C}^{q}$ with the elements $g_{1}^{k}, \ldots, g_{q}^{k}$. Each $g_{j}^{k}$ is a solution of (11) for $\epsilon=\epsilon_{k}(\mu, \nu)$ it depends on $\nu, \mu$, on the subband-index $k$ and on its lattice site $j$.

In the next section we are going to develop a method to evaluate the resolvent or Green's function $\mathbf{G}$ of $\mathbf{H}$ without explicit knowledge of the eigenvalues and eigenvectors of $\mathbf{H}$. This approach allows us to calculate an analytical expression for the diagonal elements of the resolvent in section 3, results that go beyond those obtained by Ueta in [8] In section 4 we show how to calculate the off-diagonal elements of G. A conclusion is given in section 5 .

\section{The Green's function}

The Green's function $\mathbf{G}(\lambda, \mu, \nu)$ corresponding to $\mathbf{H}(\mu, \nu)$ is defined as

$$
\mathbf{G}(\lambda, \mu, \nu)=(\lambda-\mathbf{H}(\mu, \nu))^{-1}
$$

for all $\lambda$ that are not in the spectrum of $\mathbf{H}$

If the eigenvalues $\epsilon_{k}(\mu, \nu)$ and the eigenvectors $\psi_{k}(\mu, \nu)$ of $\mathbf{H}$ were already known, $\mathbf{G}\left(\mu_{0}, \nu_{0}\right)$ could be evaluated through 9]

$$
\mathbf{G}\left(\lambda, \mu_{0}, \nu_{0}\right)=\sum_{k=1}^{q} \frac{\psi_{k}\left(\mu_{0}, \nu_{0}\right) \psi_{k}^{*}\left(\mu_{0}, \nu_{0}\right)}{\lambda-\epsilon_{k}\left(\mu_{0}, \nu_{0}\right)} .
$$

For most purposes (e.g. the calulation of impurity states) it is not sufficient to know $\mathbf{G}$ at only one point $\left(\mu_{0}, \nu_{0}\right)$ in the magnetic Brillouin zone; instead the Green's function of the original $2 \mathrm{~d}$-tight-binding Hamiltonian, i. e. over the whole spectrum of 
$\mathbf{H}$, with $\mu \in[-\pi, \pi]$ and $\nu \in[-\pi, \pi]$ is needed. This is equivalent to a summation over the entire spectrum $\left\{\epsilon_{k}(\mu, \nu)\right\}$, i.e. by integrating over $\mu$ and $\nu$ :

$$
\mathbf{G}(\lambda)=\frac{1}{4 \pi^{2}} \sum_{k=1}^{q} \int_{-\pi}^{\pi} \int_{-\pi}^{\pi} \frac{\psi_{k}(\mu, \nu) \psi_{k}^{+}(\mu, \nu)}{\lambda-\epsilon_{k}(\mu, \nu)} \mathrm{d} \nu \mathrm{d} \mu
$$

Alternatively the $(q \times q)$ matrix $\mathbf{G}(\mu, \nu)$ can be evaluated applying Cramer's rule for the inverse of a matrix:

$$
\begin{aligned}
\left(\mathbf{G}\left(\lambda, \mu_{0}, \nu_{0}\right)\right)_{m, n} & =\left((\lambda-\mathbf{H})^{-1}\right)_{m, n} \\
& =\frac{A_{m, n}}{\operatorname{det}(\lambda-\mathbf{H})} \\
& =\frac{A_{m, n}}{P(\lambda)-2 \cos \left(q \nu_{0}\right)-2 \cos \left(q \mu_{0}\right)} .
\end{aligned}
$$

For fixed values $\mu_{0}, \nu_{0} . A_{m, n}$ in (9) represents the classical adjoint of $\lambda-\mathbf{H}$ i. e. the determinant of the matrix that we get when we delete the $m$-th row and the $n$-th column of $\lambda-\mathbf{H}$, multiplied by $(-1)^{m+n}$.

As in equation (\&) we get the Green's function of the original Hamiltonian, that is for all possible values of $\mu$ and $\nu$ through integration:

$$
(\mathbf{G}(\lambda))_{m, n}=\frac{1}{4 \pi^{2}} \int_{-\pi}^{\pi} \int_{-\pi}^{\pi} \frac{A_{m, n}}{P(\lambda)-2 \cos (q \nu)-2 \cos (q \mu)} \mathrm{d} \nu \mathrm{d} \mu .
$$

In this representation $\mathbf{G}$ depends only on $\mathbf{H}$ itself and not explicitly on the eigenvalues or eigenvectors of $\mathbf{H}$.

The Green's function (10) has some really surprising features:

(i) An inspection of (4) shows that

$$
\mathbf{G}(\lambda) \in \mathbb{R}^{(q \times q)},
$$

for all $\lambda \in \mathbb{R}, \lambda \notin \operatorname{spec}(\mathbf{H})$, because the imaginary part of $A_{m, n}$, if nonzero, has always a factor $\sin (q \mu)$, so it vanishes under the integral (10).

(ii) Due to the hermiticity of $\mathbf{H}$ and (11):

$$
(\mathbf{G}(\lambda))_{m, n}=(\mathbf{G}(\lambda))_{n, m}
$$

for $\lambda \in \mathbb{R}$.

(iii) The elements in every (off-)diagonal are equal to each other:

$$
(\mathbf{G}(\lambda))_{n, n+j}=(\mathbf{G}(\lambda))_{1,1+j}
$$

for all $1 \leq n \leq q$ and $0 \leq j<q-n$. The proof of this property uses that

$$
A_{n, n+j}(\nu)=A_{1,1+j}(\nu-2 \pi \alpha n),
$$

which is easy to accept but rather lengthy to prove [10]; with this result the substitution $\nu \mapsto \nu^{\prime}:=\nu-2 \pi \alpha n$ leaves the integral (10) invariant. 


\section{The diagonal elements}

Using equations (10) to (13) we are able to calculate the diagonal elements of $\mathbf{G}$. At first we take advantage of (13):

$$
(\mathbf{G}(\lambda))_{n, n}=\frac{1}{q} \sum_{j=1}^{q}(\mathbf{G}(\lambda))_{j, j}
$$

From

$$
\frac{\mathrm{d} \operatorname{det}(\lambda-\mathbf{H}(\mu, \nu))}{\mathrm{d} \lambda}=\frac{\mathrm{d} P(\lambda)-2 \cos (q \mu)-2 \cos (q \nu)}{\mathrm{d} \lambda}=\frac{\mathrm{d} P(\lambda)}{\mathrm{d} \lambda}=\sum_{j=1}^{q} A_{j, j}
$$

the elements of (10) with $m=n$ can now be transformed to

$$
(\mathbf{G}(\lambda))_{n, n}=\frac{1}{4 \pi^{2}} \frac{1}{q} \frac{d P(\lambda)}{d \lambda} \int_{-\pi}^{\pi} \int_{-\pi}^{\pi} \frac{1}{P(\lambda)-2 \cos (q \mu)-2 \cos (q \nu)} \mathrm{d} \nu \mathrm{d} \mu .
$$

The double integral in (14) is well-known, e.g. [11], and $(\mathbf{G}(\lambda))_{n, n}$ evaluates to

$$
(\mathbf{G}(\lambda))_{n, n}=\frac{2}{\pi q} \frac{1}{P(\lambda)} \frac{\mathrm{d} P(\lambda)}{\mathrm{d} \lambda} \mathrm{K}\left(\frac{4}{P(\lambda)}\right)
$$

for all $\lambda$ not in the spectrum of $\mathbf{H}$, hence, according to (6), for $|P(\lambda)|>4$. K denotes the complete elliptic integral of the first kind, cf. [12].

In order to calculate the density of states (and other useful quantities) we need the well-known extension of $\mathbf{G}$ to complex $\lambda$ and we define

$$
\mathbf{G}^{ \pm}(\lambda)=\lim _{s \rightarrow 0} \mathbf{G}(\lambda \pm \mathrm{i} s)
$$

which is relevant for $\lambda \in \mathbb{R}$ in the continuous parts of $\operatorname{spec}(\mathbf{H})$.

The diagonal elements of $\mathbf{G}^{ \pm}(\lambda)$ are calculated with the analytic continuation of the elliptic integral [11]. For the real and the imaginary part we get

$$
\begin{aligned}
& \Re\left(\mathbf{G}^{ \pm}(\lambda)\right)_{n, n}=\operatorname{sign}(P(\lambda)) \frac{1}{2 \pi q} \frac{\mathrm{d} P(\lambda)}{\mathrm{d} \lambda} \mathrm{K}\left(\frac{P(\lambda)}{4}\right) \\
& \Im\left(\mathbf{G}^{ \pm}(\lambda)\right)_{n, n}=\mp \frac{1}{2 \pi q} \frac{\mathrm{d} P(\lambda)}{\mathrm{d} \lambda} \mathrm{K}^{\prime}\left(\frac{|P(\lambda)|}{4}\right)
\end{aligned}
$$

The density of states $\rho$ is given by the imaginary part of $\mathbf{G}^{+}$:

$$
\rho(\lambda)=-\frac{1}{\pi} \Im\left(\mathbf{G}^{+}(\lambda)\right)_{n, n}=\frac{1}{2 \pi^{2} q} \frac{\mathrm{d} P(\lambda)}{\mathrm{d} \lambda} \mathrm{K}^{\prime}\left(\frac{|P(\lambda)|}{4}\right) .
$$

This is identical to the result of Wannier, Obermair and Ray [2].

The diagonal elements of $\mathbf{G}$ and $\mathbf{G}^{+}$are shown in figure 1 . 

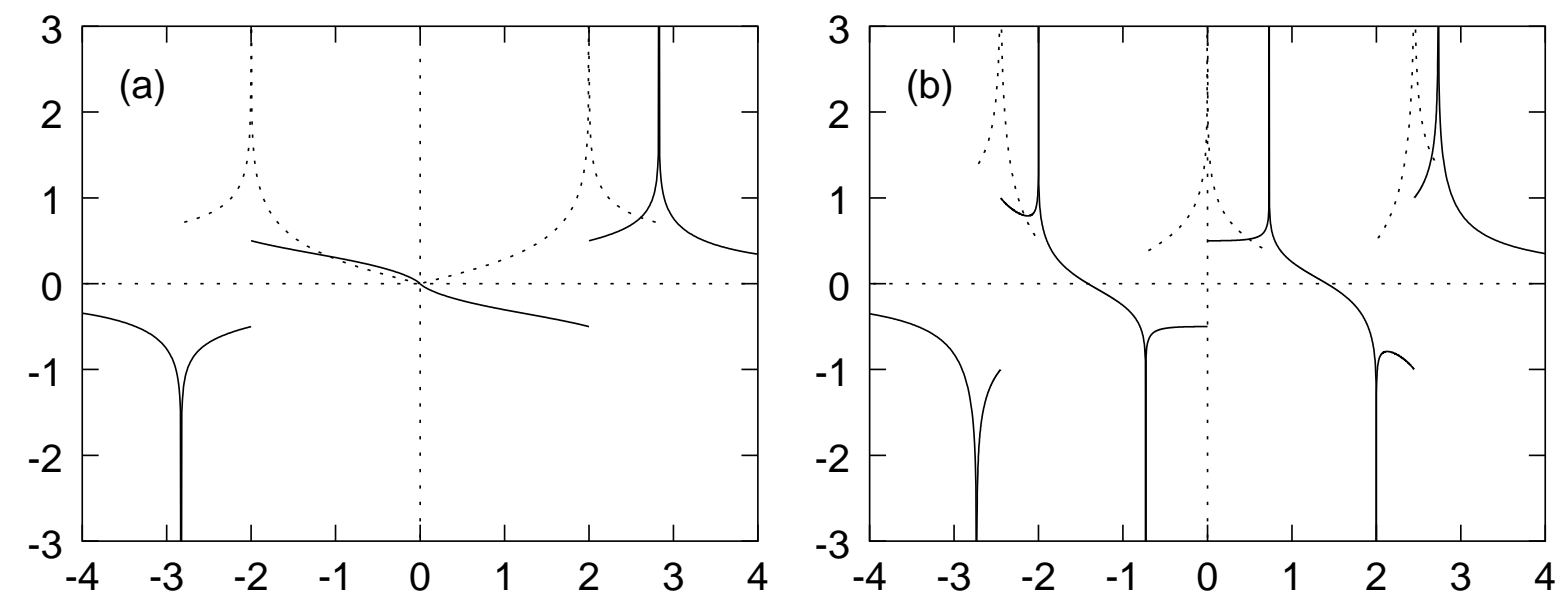

Figure 1. The diagonal elements of $\mathbf{G}(\lambda)$ and $\mathbf{G}^{+}(\lambda)$ versus $\lambda$ : real ( - ) and imaginary part (- - -) for (a) $\alpha=\frac{1}{2}$ and (b) $\alpha=\frac{1}{3}$.

\section{The Off-Diagonal Elements}

The off-diagonal elements of the Green's Function are required for many purposes, e.g. for the calculation of impurity states [13]. In the case of a vanishing magnetic field, where $P(\lambda)=\lambda$, it is possible to find a recurrence relation for all elements of the Green's Function [11]. In presence of a magnetic field we were not able to find a general recurrence. Only the elements of the first off-diagonal $(\mathbf{G}(\lambda))_{n, n \pm 1}$ may be evaluated in a similar way:

Using that

$$
(\lambda-\mathbf{H}) \mathbf{G}(\lambda, \mu, \nu)=1
$$

and

$$
\mathbf{G}(\lambda, \mu, \nu)(\lambda-\mathbf{H})=1
$$

for every $\mu$ and $\nu$, we get after integration

$$
\begin{aligned}
& (\mathbf{G}(\lambda))_{i+1, j}+(\mathbf{G}(\lambda))_{i-1, j}+(\mathbf{G}(\lambda))_{i, j+1}+(\mathbf{G}(\lambda))_{i, j-1}= \\
& =\frac{1}{4 \pi^{2}} \int_{-\pi}^{\pi} \int_{-\pi}^{\pi}\left(2 \lambda-c_{i}-c_{j}\right) \frac{A_{i, j}}{P(\lambda)-2 \cos (q \mu)-2 \cos (q \nu)} \mathrm{d} \mu \mathrm{d} \nu-2 \delta_{i, j} .
\end{aligned}
$$

With (12) we get an expression for the elements of the first off-diagonal:

$$
\begin{aligned}
& (\mathbf{G}(\lambda))_{i, i+1}= \\
& =\frac{1}{2}\left(\frac{1}{4 \pi^{2}} \int_{-\pi}^{\pi} \int_{-\pi}^{\pi}\left(\lambda-c_{i}\right) \frac{A_{i, i}}{P(\lambda)-2 \cos (q \mu)-2 \cos (q \nu)} \mathrm{d} \mu \mathrm{d} \nu-1\right) .
\end{aligned}
$$

The integral in (17) yields [10]

$$
(\mathbf{G}(\lambda))_{i, i+1}=\frac{1}{4}\left(\lambda(\mathbf{G}(\lambda))_{i, i}-1\right) .
$$


All the other elements of the Green's Function have to be calculated numerically by integrating (10), the result for $\alpha=\frac{1}{5}$ is shown in figure 2 .
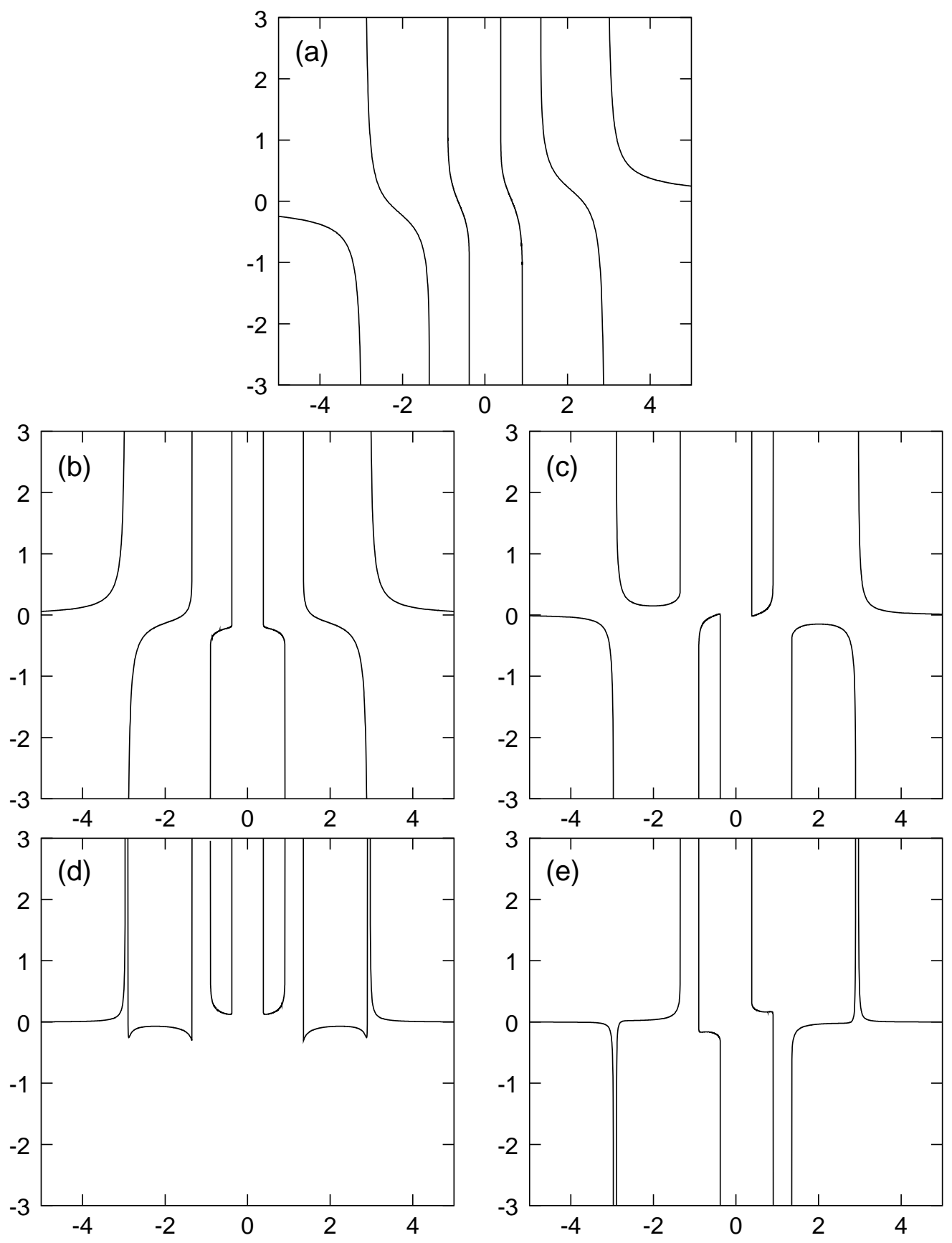

Figure 2. All different elements of $\mathbf{G}$ for $\alpha=\frac{1}{5}:(\mathbf{G}(\lambda))_{n, n+l}$ versus $\lambda$ with $l=0$ (a), $l=1(\mathrm{~b}), l=2(\mathrm{c}), l=3(\mathrm{~d})$ and $l=4(\mathrm{e})$.

For some purposes it ist necessary to extend the Green's Function over more than one magnetic period in $x$-direction $(|m-n|>q)$. For this case a continuation of $\mathbf{G}$ can by constructed by applying equation (2) to equation (7). The elements of this 
continuation are given by

$$
(\mathbf{G}(\lambda))_{m, n}=\frac{1}{4 \pi^{2}} \int_{-\pi}^{\pi} \int_{-\pi}^{\pi} \mathrm{e}^{\mathrm{i} q \mu h} \frac{A_{m, n^{\prime}}}{P(\lambda)-2 \cos (q \nu)-2 \cos (q \nu)} \mathrm{d} \nu \mathrm{d} \mu
$$

where $h$ is the integer part of $(m-n) / q$ and $n^{\prime}$ the remainder of $n / q$.

For each fixed $n^{\prime} \in[0, q-1]$ we see in figure 3, with $\alpha=\frac{1}{3}$ as an example, that $\mathbf{G}\left(\lambda, m, n^{\prime}+k q\right)$ decreases exponentially with growing $|m-n|$ (see figure 3 ).

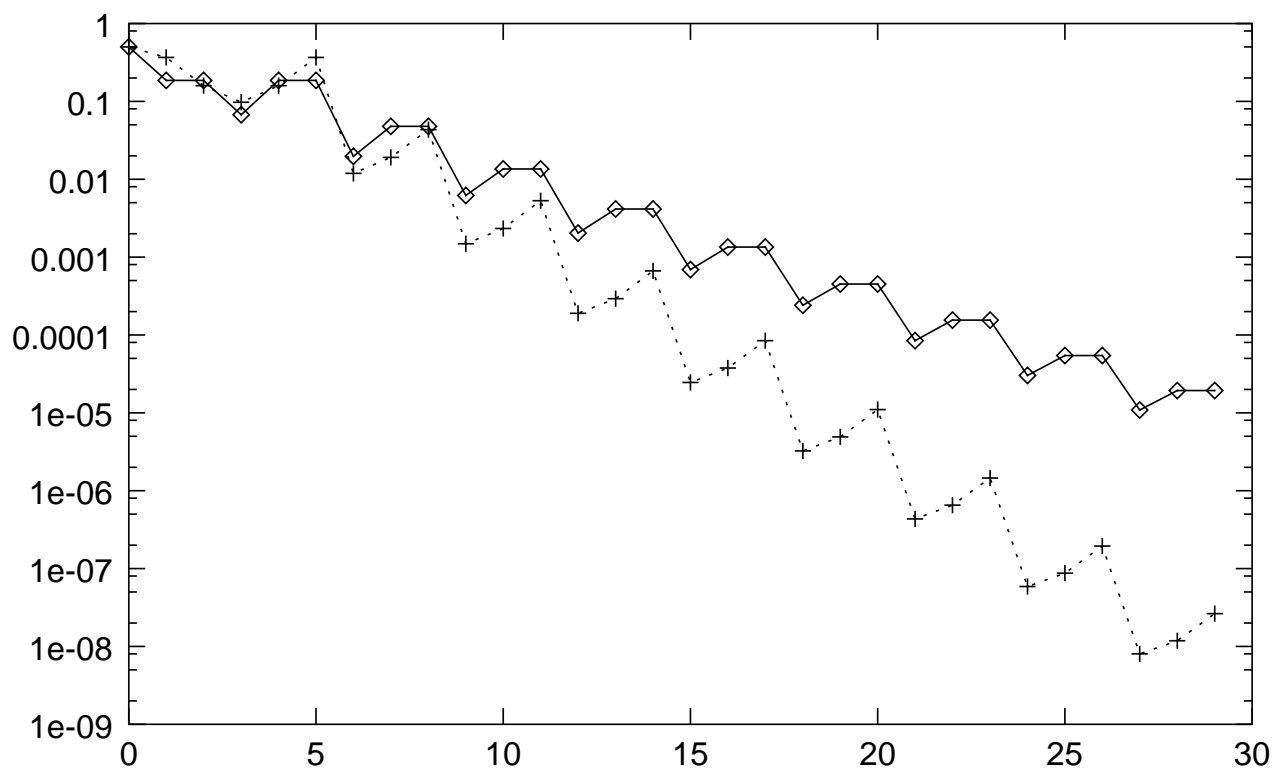

Figure 3. The extended Green's Function $(\mathbf{G}(\lambda))_{m, n}$ (see equation (18)) for $\alpha=\frac{1}{3}$ versus $|m-n|: \lambda=1.9(\diamond)$ and $\lambda=-3.4(+)$, scaled logarithmically. The lines only serve as a guide to the eye.

\section{Conclusion}

In the case of a vanishing magnetic field, $\alpha=0, P(\lambda)$ reduces to

$$
P(\lambda)=\lambda \text {. }
$$

If we put this into the equations (15) to (16), we get the diagonal elements of the Green's Function of the Tight Binding Hamiltonian as calculated in [11]. Thus our results are consistent in the limit $\alpha=0$.

From another point of view, we could say, that the minimal coupling to the vector potential

$$
\hat{p} \mapsto \hat{p}+e \vec{A},
$$

that is used to obtain the almost-Mathieu equation for electrons in the one-band-model, is, within this model, equivalent to the transformation

$$
\lambda \mapsto P(\lambda) .
$$


In a forthcoming paper [13] the results will be used to solve Dyson's equation for a model with isolated impurities, i.e. to calculate impurity states superimposed on Hofstadter's butterfly.

\section{References}

[1] Hofstadter D R, "The energy-levels of bloch electrons in rational and irrational magnetic fields", Phys. Rev. B 15 (1976), 6.

[2] Wannier G H, Obermair G M and Ray R, "Magnetoelectric density of states for a model crystal", Phys. Stat. Sol. (b) 93 (1979), 337-342.

[3] Belissard J and Simon B, "Cantor spectrum for the almost Mathieu equation", J. Funct. Anal. 48 (1982), 408-419.

[4] Thouless D J, "Total bandwidth for the Harper equation: III", J. Phys. A: Math. Gen. 24 (1991), 4055-66.

[5] Herrmann D J L and Janssen T, "On spectral properties of Harper-like models", J. Math. Phys. 40 (1999), 1197-1214.

[6] Thouless D J, Kohmoto M, Nightingale M P and den-Nijs M, "Quantized Hall conductance in a two-dimensional periodic potential", Phys. Rev. Let. 49 (1982), 405-408.

[7] Albrecht C, Smet J M, von Klitzing K, Weiss D, Umansky V and Schweizer H, "Evidence of Hofstadter's fractal energy spectrum in the quantized Hall conductance", Phys. Rev. Let. 86 (2001), 147-150.

[8] Ueta T, "Lattice Green function in uniform magnetic fields", J. Phys. A: Math. Gen. 30 (1997), 5339-5350.

[9] Economou E N, "Green's Function in Quantum Physics" (Springer Berlin, Heidelberg, New York), 1979.

[10] Chmela F G, "Störstellen auf Hofstadters Schmetterling oder: Zur Greensfunktion des almost-Mathieu-Operators", Ph. D. thesis (in German), University of Regensburg, 2000. (http://www.bibliothek.uni-regensburg.de/opus/volltexte/2001/28)

[11] Morita T, "Useful procedure for computing the lattice Green's function - square, tetragonal and bcc lattices", J. Math. Phys. 13 (1971), 1744.

[12] Byrd P, Friedman M, "Handbook of Elliptic Integrals for Engineers and Scientists" (Springer Berlin), 1971.

[13] Obermair G M, Chmela F G, to be published. 